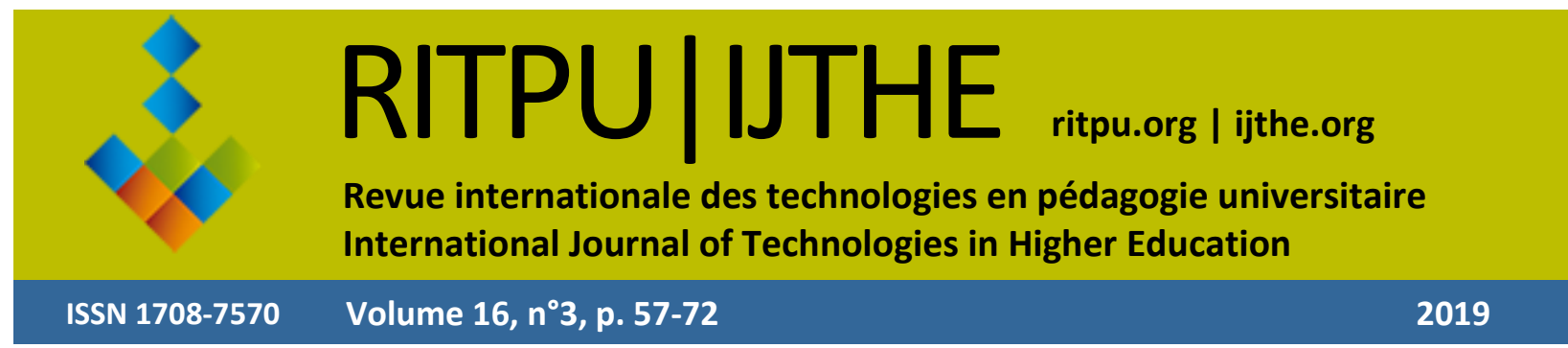

\title{
La gestion des tuteurs en ligne, pour un tutorat de qualité
}

Managing distance education tutors online: Towards improving online learning

https://doi.org/10.18162/ritpu-2019-v16n3-04

\author{
Nicole RACETTE \\ Université TÉLUQ, Canada \\ racette.nicole@teluq.ca \\ Guillaume DESJARDINS \\ Université TÉLUQ, Canada \\ guillaume.desjardins@teluq.ca \\ Marie-Pierre BOURDAGES-SYLVAIN \\ Université TÉLUQ, Canada \\ mbourdag@teluq.uquebec.ca \\ Martin HOULE \\ Université TÉLUQ, Canada \\ martin.houle@teluq.ca
}

\section{Résumé}

Cet article examine la question de recherche suivante: Afin d'assurer une bonne qualité d'encadrement dans les cours universitaires en ligne, selon les écrits scientifiques, quels types de compétences doivent être développés, d'une part, chez les tuteurs et, d'autre part, chez les gestionnaires de ces tuteurs? Des compétences en encadrement sont nécessaires, mais également en gestion du travail, en échange social et surtout dans l'utilisation des TIC dans un contexte de télétravail, et ce, afin de maintenir la motivation et l'efficacité du travail.

\section{Mots-clés}

Formation en ligne, tuteurs, télétravail, encadrement, compétences, gestion, transfert de connaissances, numérique

\section{Abstract}

This article examines the following research question: In order to ensure a good quality of supervision in online university courses, according to the scientific literature, what types of skills should be developed among tutors, on the one hand, and among the managers of these tutors, on the other hand. Management skills are required, but also in work organization, social exchange, especially in a teleworking context, in the use of ICT, in order to maintain motivation and efficiency of work.

\section{Keywords}

Online training, tutors, teleworking, supervision, skills, management, knowledge transfer, digital 


\section{Introduction}

Le numérique a permis une offre importante de cours en ligne, mais il a également mis à l'épreuve les pratiques dans l'enseignement, entre autres, par rapport à l'introduction d'un nouveau corps d'emploi : les tuteurs. L'angle sous lequel nous traitons de la formation en ligne dans cet article se rapporte au tutorat dans les cours universitaires crédités offerts totalement en ligne. Dans ce contexte, nous examinons la situation des tuteurs dans leurs actions et dans la gestion de ces actions, personnel normalement absent des MOOC (massive open online courses), aussi appelés FLOT (formation en ligne ouverte à tous) ou CLOM (cours en ligne ouverts aux masses) (Conseil supérieur de l'éducation [CSE], 2015). Ainsi, nous considérons le travail du tuteur comme « une forme d'aide individualisée, offerte soit pour accompagner un apprenant qui éprouve des difficultés, soit pour donner une formation particulière, complémentaire » (« Tutorat », 2003).

Dans les cours universitaires crédités offerts en ligne, l'encadrement est le plus souvent dissocié de l'enseignement (Béché et Schneider, 2019), et ce, surtout lorsque le nombre d'inscriptions dépasse le nombre d'étudiants qu'un enseignant peut encadrer lui-même. Dans la plupart des cas, ces tuteurs sont gérés par des enseignants ou des spécialistes en éducation (Racette et BourdagesSylvain, 2019). Ils sont normalement embauchés à forfait pour la durée du cours de chaque étudiant qu'ils ont sous leur responsabilité. Des écrits scientifiques ont largement fait état des compétences nécessaires pour faire un bon tutorat (Borges, Coelho, Faiad et Rocha, 2014; Brindley, 2014; De Metz et Bezuidenhout, 2018; Goodyear, Salmon, Spector, Steeples et Tickner, 2001). Toutefois, peu de recherches tiennent compte du contexte de travail et des pratiques de gestion de ce travail. Les recherches à ce propos sont rares, probablement en raison de cette interdisciplinarité entre le tutorat et la gestion du tutorat, qui se côtoient rarement dans leur aspect systémique.

La question de recherche de notre étude, financée par le Fonds de recherche du Québec - Société et culture (FRQSC), est la suivante : Afin d'assurer une bonne qualité d'encadrement dans les cours universitaires en ligne, selon les écrits scientifiques, quels types de compétences doivent être développés, d'une part, chez les tuteurs et, d'autre part, chez les gestionnaires de ces tuteurs? Cet article devrait permettre de sensibiliser les établissements d'enseignement universitaire qui offrent des cours en ligne sur les façons de faire et sur celles qui sont à proscrire dans la pratique du tutorat, mais surtout, dans les pratiques de gestion de ce personnel. Il permettra également d'élargir la compréhension scientifique du management en formation en ligne. À partir des écrits scientifiques, nous présentons tout d'abord le contexte de travail de l'enseignement en ligne et la forme de gestion des tuteurs qu'on y retrouve ou qu'on devrait y retrouver. Finalement, nous présentons les pratiques et compétences relevées dans les écrits scientifiques qui semblent essentielles, chez les tuteurs et chez leurs supérieurs, afin d'assurer un encadrement de qualité dans les cours universitaires en ligne.

\section{Le contexte de travail dans les cours en ligne}

Plusieurs catégories de spécialistes interviennent dans les cours en ligne, par exemple pour les tâches de conception ou de diffusion des cours, d'encadrement des étudiants et de suivis des cours (Béché et Schneider, 2019; Depover, 2013; Heiz, 2011; Pudelko, 2017; Roy, Poellhuber et Lefebvre, 2017), et ce, contrairement aux cours en présentiel où ce sont les enseignants qui assument habituellement l'entièreté de ces tâches. Dans les cours en ligne, l'enseignant, ou le concepteur embauché à forfait définit le contenu du cours, les objectifs et les activités d'apprentissage, et ce, souvent en collaboration avec un spécialiste en éducation (Lédé et 
Pelissier, 2019). C'est aussi l'enseignant, ou le concepteur à forfait, qui conçoit normalement les activités d'évaluation, bien qu'il s'agisse parfois d'une tâche déléguée à un spécialiste en éducation. Toutefois, la mise en ligne des cours est faite par d'autres spécialistes, entre autres en ce qui a trait à l'audiovisuel, au graphisme et à l'informatique. Étant donné que les cours offerts en ligne peuvent accueillir un gros volume d'étudiants, c'est aussi un autre spécialiste, appelé tuteur, correcteur, encadrant, personne-ressource, etc., qui assume leur encadrement. Cette personne travaille normalement à son domicile, loin des autres corps d'emploi de l'établissement.

Parce qu'en formation en ligne, le travail d'enseignement est morcelé, contrairement à l'enseignement sur campus, et que l'encadrement des étudiants est souvent fait par une autre personne que celle qui a conçu le cours, il y a des risques d'erreurs, d'incohérence ou de distorsion dans les messages livrés aux étudiants, nécessitant que des procédures soient soigneusement établies et suivies par les différents intervenants (Depover, 2013). Dans ce contexte relativement nouveau dans le monde de l'éducation, il semble essentiel que les tuteurs développent des compétences particulières dans leur travail et par rapport à leur contexte de travail (Brindley, 2014; Heuel et Feldmann, 2014). Malheureusement, leur fonction est souvent mal définie, peu reconnue, en plus de se pratiquer à domicile, loin des collègues de travail. Pour que ce travail se déroule bien, les gestionnaires des tuteurs doivent développer des compétences particulières également par rapport à cette gestion, incluant la gestion du télétravail. Baret et Oiry (2014) définissent la compétence comme étant un savoir-agir par rapport à différentes situations. Ces derniers auteurs précisent que les compétences individuelles et collectives se construisent grâce à la possibilité de faire face à des événements imprévisibles dans le travail et d'y répondre efficacement. Pour l'instant, le tutorat et la gestion de ce tutorat semblent se pratiquer selon les compétences que chacun possède déjà naturellement, sans que des formations spécifiques soient exigées. De plus, les établissements semblent être des organisations peu apprenantes, dans ce sens que les apprentissages verticaux (par rapport au passé) et les apprentissages horizontaux (par rapport aux collègues) sont peu mis à contribution dans le travail de chacun (Landry et Gril, 2017).

\section{La gestion des tuteurs}

Bien que plusieurs recherches portent sur les modes d'enseignement en ligne et sur les modèles de tutorat, les différentes composantes de la formation en ligne sont rarement abordées dans une perspective systémique, bien que l'articulation de ces composantes demeure une préoccupation importante à travers le Canada (CSE, 2015). Selon Racette, Poellhuber et Bourdages-Sylvain (2018), la relation des tuteurs avec les équipes pédagogiques est éloignée à bien des points de vue, ce qui nuit à la qualité des cours offerts. Ainsi, la gestion des tuteurs est confiée, dans la plupart des cas, à l'enseignant d'un cours ou à un spécialiste en éducation. La connaissance fine des cours et des solutions qui peuvent être apportées aux problèmes susceptibles de se présenter concerne davantage les enseignants. Mais, ces derniers sont d'abord des spécialistes de contenu, et non des technologies ou de la gestion du personnel. Concernant les spécialistes de l'éducation, ils ne sont ni spécialistes de contenu ni spécialistes de la gestion du personnel. Dans un cas comme dans l'autre, on retrouve des obstacles à assurer une bonne gestion des tuteurs. Cette situation se complexifie également du fait qu'un même tuteur peut être géré par plusieurs responsables. La figure 1 témoigne de cette complexité quant à l'encadrement du travail des tuteurs. 


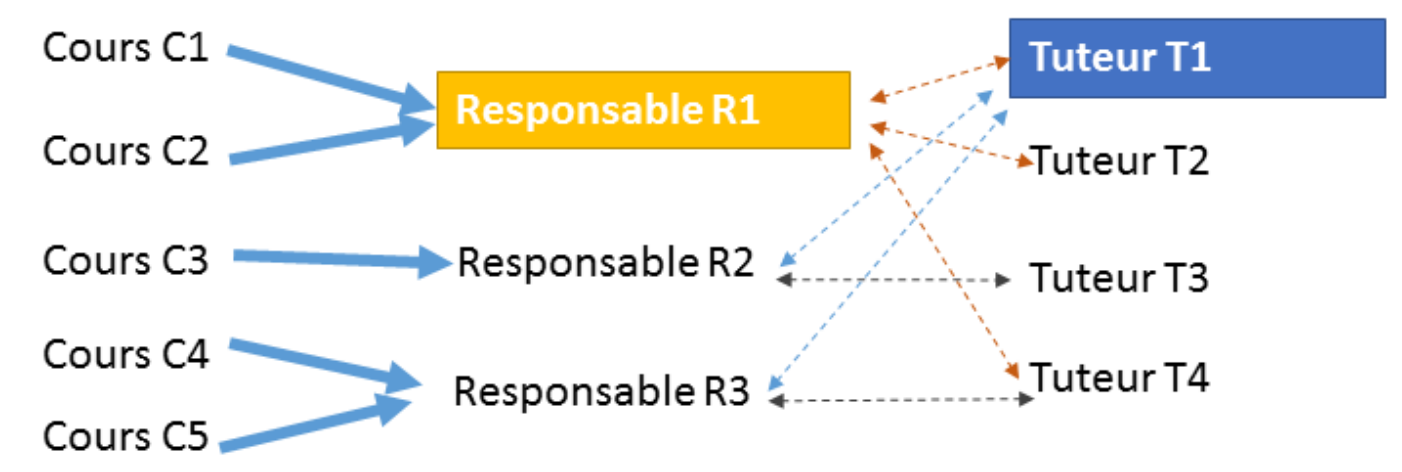

Figure 1

La complexité de la gestion des tuteurs par leurs responsables

Comme l'indiquent ces lignes, les tuteurs travaillent souvent dans plusieurs cours différents, avec différents responsables de cours, chacun ayant ses exigences, sa façon de travailler et sa façon de communiquer, ce qui complique leur travail (Racette et al., 2018). Dans cette figure, le responsable R1 doit gérer les tuteurs T1, T2 et T4 par rapport à deux cours, C1 et C2. Et le tuteur $\mathrm{T} 1$ doit se rapporter aux responsables R1, R2 et R3 par rapport à au moins trois cours, démontrant ainsi la complexité de cette gestion (Depover, 2013; Heiz, 2011). Les liens entre un cours et le responsable des tuteurs de ce cours sont forts (représentés par une ligne épaisse et continue) puisque le responsable est normalement l'enseignant lui-même ou le spécialiste en éducation qui a travaillé à concevoir le cours. Toutefois, les liens entre le responsable et les tuteurs qui travaillent à l'encadrement des étudiants dans le cadre d'un cours sont plutôt faibles (représentés par des lignes étroites et pointillées). Ainsi, ce contexte de travail empêche les responsables des tuteurs d'avoir une vue d'ensemble du travail réalisé par chacun des tuteurs et, parce que ces derniers travaillent à leur domicile, la création et le transfert des connaissances s'effectuent rarement ou très peu (Taskin, 2006). Une zone d'inconfort existe relativement à cette gestion du travail des tuteurs, surtout dans un contexte de télétravail.

Du fait que les tuteurs travaillent à partir de leur domicile, ils vivent des difficultés particulières. Ils ont peu accès à leurs collègues enseignants et tuteurs. De plus, ils travaillent normalement à temps partiel et sans garantie d'embauche d'une session à l'autre (Racette et al., 2018). Leur travail consiste à répondre aux questions des étudiants, la semaine comme la fin de semaine, selon les paramètres qu'ils se fixent eux-mêmes, et ce, en grande partie au moyen de courriels (Pudelko, 2017). Ils font un travail plutôt invisible qui consiste essentiellement à envoyer des courriels, à répondre au téléphone, à corriger des travaux et des examens et, dans de rares occasions, à intervenir dans un forum ou dans des visioconférences (Marcotte, 2019; Pudelko, 2017; Racette et al., 2018). Ce travail invisible s'échelonne sur plusieurs mois par rapport à l'encadrement d'un même étudiant, ce qui rend difficile l'évaluation régulière de ce travail. Par exemple, un professeur relatait un cas particulier où la direction de l'établissement avait été avertie par un étudiant qu'il n'avait reçu aucun courriel, aucun travail ou examen corrigé de son tuteur, et ce, depuis le début du cours trois mois auparavant. Pourtant, ce tuteur se voyait rémunéré depuis le début du cours. Il semble donc évident que ce long échéancier dans l'encadrement d'un étudiant et cette invisibilité dans le travail des tuteurs peuvent dissimuler des abus de la part de ces derniers, mais également un manque de reconnaissance de leur travail de la part de leur supérieur. Pour éclairer les pratiques actuelles dans la gestion du travail des tuteurs, dans les lignes qui suivent, nous présentons quelques écrits scientifiques par rapport à la gestion, à la communication en ligne et, finalement, au télétravail. 


\subsection{Des principes de gestion}

Il va de soi qu'une bonne gestion mène à la construction de compétences, à une efficacité dans le travail ainsi qu'à la motivation et à l'engagement des employés. Pour assurer une qualité de gestion, selon Livian (2010), plusieurs mauvaises pratiques sont à éviter. Sur le plan individuel, il s'agit de l'inadéquation formation-emploi, alors que sur le plan collectif, ce sont plutôt les conditions de travail inadaptées et les animations d'équipes insuffisantes qui sont à proscrire. Pour ce qui est du plan organisationnel, les changements fréquents non préparés, l'adoption rapide de technologies mal assimilées, une mauvaise gestion des carrières, des procédures inadaptées ou inappropriées, la monopolisation du savoir et la sous-traitance en cascade sont les pièges qui guettent les gestionnaires. Comme la gestion dans les cours en ligne est relativement nouvelle dans nos établissements postsecondaires au Québec, et compte tenu du contexte de télétravail qui prévaut chez les tuteurs, ces embûches semblent prendre une grande importance.

En ce qui concerne les qualifications du personnel, selon Baret et Oiry (2014), il faut éviter deux types d'incompétence pouvant être dommageables autant pour les usagers et les employés que pour l'organisation dans son ensemble : l'incompétence organisée et l'incompétence diplômée. L'incompétence organisée est produite, en partie, par les mauvaises pratiques présentées plus haut. Des changements trop fréquents, sans formation adéquate, laissent les employés sceptiques quant aux résultats espérés et déçus de l'organisation relativement à la maîtrise des opérations. L'incompétence organisée peut également résulter de conditions de travail inadaptées dans lesquelles l'employé sait qu'il lui est impossible de réaliser correctement le travail demandé, n'ayant ni le temps, ni les moyens, ni même la rémunération nécessaires. Malheureusement, il s'agit ici de problèmes formulés par plusieurs tuteurs dans l'étude de Racette, Poellhuber et Bourdages-Sylvain (2017, p. 10-11) : «Les tuteurs expliquent que leur tâche est de plus en plus exigeante et que cette tâche réclame de nouvelles compétences (animation, gestion de groupe, compétences informatiques, gestion des courriels, visioconférences, etc.), mais qui, pour le moment, ne s'accompagnent pas d'une reconnaissance professionnelle ni financière suffisante. » Cette incompétence organisée peut également résulter d'une confiance exagérée dans certains outils ou procédures qui déresponsabilise les employés devant les problèmes à régler, se voyant dans l'impossibilité de s'écarter du système mis en place. Le morcellement du travail dans l'enseignement en ligne crée le problème de la main gauche qui ne sait pas ce que fait la main droite. Il y a également un danger de multiplier des normes contradictoires.

D'un autre côté, l'incompétence diplômée résulte de repères professionnels insuffisants qui se manifestent par un manque de compréhension des compétences requises, un apprentissage trop superficiel, une mauvaise intégration des règles de qualité, de mauvais conseils de mentors et peu ou pas de communauté de pratique. Ils se manifestent également par des routines où les salariés ne prennent plus le recul nécessaire par rapport au travail à faire et ne font que reproduire ce qui se fait habituellement (Livian, 2010). Dans ce cas, il y a perte de sens critique ou, encore, évitement de conflits qui pourraient occasionner un questionnement. Pour assurer une cohérence dans le travail de tutorat, il faut prendre le temps de bien agir. Entre autres, comme le rapporte Rosa (2019): «Le renouvellement fréquent des plateformes informatiques (courriels, applications pédagogiques, communications internes, etc.) exige des périodes de formation et un temps d'intégration. »

Les qualités nécessaires aux gestionnaires des tuteurs, pour rendre le travail de tutorat efficace, semblent manquer, probablement en raison de leur formation inadéquate, mais également de décisions de la part des établissements qui manquent de cohérence relativement aux pratiques de chacun. Comme 1'explique Dupuich (2011, p. 114) : «Tout le talent d'un manager est de savoir 
mettre en exergue les talents individuels et de faire converger ces talents en connaissances collectives: c'est-à-dire susciter les liens entre les expertises de chacun et favoriser les synergies. »

\subsection{Des principes de communication en ligne}

Force est de constater que la communication numérique, utilisée dans le télétravail, constitue une limite à l'échange social. La communication, essentiellement en ligne, entre les responsables des tuteurs et les tuteurs empêche la création d'un climat social propice à la coopération. Elle limite le développement des métaconnaissances sur l'autre et le sentiment d'appartenance. Cette situation favorise des stratégies de défense psychologique, par peur de faire des erreurs qui lui seraient reprochées, ainsi qu'un manque d'occasions d'ajustements relationnelles, entraînant une détresse et un sentiment d'isolement (Enel, 2016). Le manque d'échange social maintient donc les situations de dysfonctionnement. La qualité de vie au travail est perturbée par un stress plus important, une dépersonnalisation des individus et un aplatissement des hiérarchies (Boboc et Taboy, 2014; De la Rupelle et al., 2015). L'Observatoire de la responsabilité sociétale des entreprises (De la Rupelle et al., 2015) mentionne que dans les échanges numériques, il y a un danger que s'installe une familiarité trop grande pouvant être interprétée comme un manque de respect de la part du destinataire. Selon l'ORSE, ce sont les pratiques numériques quotidiennes qui créent de bonnes ou de mauvaises habitudes et affectent positivement ou négativement ces variables. Pour améliorer le lien social en contexte de télétravail, il suggère d'instaurer une «machine à café virtuelle » pour permettre les conversations informelles, ou encore, des plateformes de discussion sur des thèmes particuliers. Ainsi, les outils de communication numériques permettent de développer une logique collective plutôt qu'individuelle, mais risquent de masquer cette dimension relationnelle nécessaire à une intelligence collective et de désengager les participants du projet dans son ensemble (Enel, 2016; Imhoff et Silva, 2016). Pour que les gens aient envie de partager leurs sentiments et leurs idées, un climat de confiance doit prévaloir. L'information doit avoir du sens, et c'est la qualité de la relation qui lui en donne, soit l'estime, la confiance, la valorisation et le respect réciproque (Kourilsky, 2014). Selon cet auteur (p. 100) : «Une communication de haute qualité s'évalue au confort, au bien-être et au plaisir qu'elle apporte. " Afin d'éviter les attaques et de favoriser l'enrichissement des équipes, la communication doit faire ressortir les différences dans les perceptions et les désirs de chacun, favorisant la construction du «nous » plutôt que du «je », ou encore, le «vous » qui sépare (Fisher, 1981; Kourilsky, 2014). Par exemple, l'accord sur le développement de la qualité de vie au travail présenté par le groupe AREVA en France, le 31 mai 2012, précise que les parties :

... soulignent la nécessité de veiller à ce que [1'usage des Nouvelles Technologies de l'Information et de la Communication .... respecte la qualité du lien social au sein des équipes et ne devienne pas un facteur conduisant à l'isolement des salariés sur leur lieu de travail, garantisse le maintien d'une relation de qualité et de respect du salarié tant sur le fond que sur la forme de la communication, ne devienne pas un mode exclusif d'animation managériale, et de transmission des consignes de travail... (p. 16).

Ces mises en garde nous semblent pertinentes pour éviter les inefficacités au travail, mais surtout, la détresse psychologique des travailleurs. À ce propos, Roman (2010) distingue les discours «bem-ditos» et les «mal-ditos»; les premiers sont produits dans la sphère administrative et visent une finalité, alors que les seconds sont plutôt produits dans la sphère personnelle et affective et ne poursuivent aucune finalité autre que le plaisir ou le défoulement. 
Idéalement, les deux types de discours devraient être présents dans une organisation, puisque la gravité administrative inhibe l'émotion traduisant un besoin de communiquer de façon informelle et sans trop de censure (Enel, 2016; Roman, 2010). Pour que ces discours «malditos » existent, il doit y avoir une grande confiance entre les employés.

\subsection{Des principes de gestion du télétravail}

Le télétravail tel que le vivent normalement les tuteurs comporte ses avantages, mais également de graves inconvénients, autant pour les tuteurs eux-mêmes que pour l'employeur. Lorsqu'il est pratiqué à cent pour cent, c'est-à-dire lorsque l'employé n'a pas de bureau de travail à l'établissement et ne s'y rend pratiquement jamais pour rencontrer ses collègues ou supérieurs, il y a alors lieu de porter une attention particulière aux écueils qui ne manquent pas de se présenter pour les employés, pour l'organisation et dans l'organisation du travail.

\subsubsection{Les impacts pour l'employé}

Le travail à domicile, que permettent de plus en plus les technologies, fait référence à 1) la liberté de lieu, séparant les télétravailleurs des autres employés de l'organisation, 2) la liberté de temps, et 3) la liberté d'action (Taskin, 2006, 2010). Des auteurs soulignent les avantages suivants pour les télétravailleurs (Enel, 2016; Fonner et Roloff, 2010; Gajendra et Harrison, 2007; Ismail, Hamsa et Mohamed, 2016; Taskin et Tremblay, 2010) : la conciliation travailfamille; le confort du travail à la maison; les gains de temps et d'argent (habillement, repas à l'extérieur, transport); la flexibilité d'horaire et de lieu; la flexibilité dans la façon de travailler; la réduction des interruptions de l'activité professionnelle et les distractions moins importantes. Toutefois, selon Taskin et Tremblay (2010), le télétravail a un effet négatif sur l'identité sociale de l'individu, surtout lorsqu'il lui est imposé. Les télétravailleurs, exerçant leurs activités professionnelles ailleurs qu'au bureau, décident du quand et du comment ils travailleront, mais perdent les frontières de la vie professionnelle et de la vie familiale (Marcotte, 2019; McDowall et Kinman, 2017; Taskin, 2006; Taskin et Tremblay, 2010). De plus, ils travaillent dans une distance psychosociologique par rapport aux collègues, puisqu'ils sont loin des espaces communs, des échanges formels ou informels, ainsi que dans une distance sur le plan de la culture organisationnelle. Ils ont peu de contact social, ce qui peut entraîner un sentiment d'isolement professionnel et social susceptible de nuire à la productivité (Golden, Veiga et Dino, 2008), voire de détruire le lien social (Fernandez, Guillot et Marrauld, 2014). De plus, le manque de reconnaissance des collègues qui travaillent à l'établissement (Enel, 2016; Fernandez et al., 2014) et leur travail invisible entrainent un déficit de valorisation et de possibilité de corriger les mauvaises pratiques (Pudelko, 2017; Racette et al., 2017), une réduction du transfert de connaissances (Beauregard, Basile et Canónico, 2019; Taskin et Bridoux, 2010) ainsi que des effets pervers en matière d'augmentation de la charge de travail (Fernandez et al., 2014). Certains télétravailleurs craignent de ne pas être considérés pour les promotions (McCloskey et Igbaria, 2003). De plus, les dysfonctionnements des outils technologiques (Enel, 2016; Tustin, 2014) et les difficultés à obtenir de l'aide de collègues (Enel, 2016; Fernandez et al., 2014) génèrent du stress (Barros, 2017). La gestion par leurs supérieurs (enseignants ou spécialistes en éducation) vise ainsi le court terme plutôt que la gestion de carrière (Taskin, 2006). Dans le télétravail, l'individu éloigné est ignoré par rapport à ses penchants, ses émotions et ses idéologies. Les politiques formelles sont souvent insuffisantes. Cette distance physique occasionne une absence de soutien et de compréhension ainsi qu'un impact négatif sur les aspects sociaux et émotionnels liés aux interactions, qui détermineront l'implication de l'employé, sa loyauté, la qualité de ses interactions, sa satisfaction au travail et son sentiment 
d'isolement. De plus, la flexibilité qu'apporte le télétravail aux employés serait une source d'intensification et de précarisation du travail (Largier, 2001; Marcotte, 2019; Taskin, 2006). Il s'agit là de commentaires recueillis chez les tuteurs dans l'étude de Racette et al. (2017). Pour développer la motivation et l'autoefficacité au travail, comme le mentionne Carré (2004/5), la dimension affective de l'individu est fondamentale pour lui, mais également pour le bon fonctionnement de l'organisation.

\subsubsection{Les impacts pour l'organisation}

Le télétravail est intéressant financièrement pour les organisations puisqu'aucun bureau n'est nécessaire pour l'employé et que de plus, il se traduit normalement par une hausse de leur productivité (Bloom, Liang, Roberts et Ying, 2014; Enel, 2016; Fernandez et al., 2014). Il y a de grandes difficultés à contrôler le télétravail, puisque le contrôle ne peut s'exercer que sur le visible, c'est-à-dire sur les résultats, ignorant souvent les aspects qualitatifs et contextuels (Taskin et Tremblay, 2010). De plus, plusieurs études évoquent l'existence d'une relation en « $\mathrm{u}$ », inversée quant aux bénéfices du télétravail, caractérisée par une diminution de la satisfaction au travail à mesure que le temps de télétravail augmente (Feldman et Gainey, 1998; Golden, 2006; Golden et Veiga, 2005). Lorsque le télétravail est effectué à temps partiel, ses aspects négatifs, tels que l'isolement social et professionnel, seraient compensés par ses avantages, notamment ceux qui sont liés à l'autonomie et à la conciliation des impératifs professionnels et familiaux. Au contraire, lorsque le travail à distance est effectué à temps plein, ses effets négatifs tendent à miner la satisfaction qu'en retire le travailleur. Plus l'entièreté du travail se fait à distance de l'établissement, plus le risque est grand de connaître une diminution de l'implication et de la loyauté de l'employé (Fernandez et al., 2014). Selon Baruch et Nicholson (1997), le contrôle managérial doit permettre aux responsables de s'assurer que les décisions et comportements développés par les télétravailleurs sont en cohérence avec les objectifs de l'organisation (Enel, 2016). Mais, un tel contrôle entre en conflit avec l'autonomie recherchée dans ce contexte de travail. La proportion de télétravail représente donc l'un des principaux effets modérateurs des aspects négatifs associés au télétravail (Beauregard et al., 2019), comme le montre la recherche réalisée auprès de travailleurs totalement à distance, dans une agence de placement de personnel. Le contrôle exercé sur les télétravailleurs se faisait à l'aide d'un agenda partagé, de rapports d'activités journaliers et d'une politique de formation de 35 jours par an. La gestion axée sur le court terme impliquait un manque d'observation sur le travail global de l'employé, ce qui avait une incidence sur le développement de sa carrière (Taskin, 2006). L'acte prenait alors plus d'importance que l'acteur, qui se voyait surtout confier des tâches techniques, répétitives et surveillées à la trace. La communication des télétravailleurs avec les succursales comptait de nombreux problèmes de partage d'informations, un manque de détails dans les informations transmises ainsi qu'une mauvaise interprétation des consignes, ce qu'on retrouve également dans le contexte de travail des tuteurs (Racette, Poellhuber et Bourdages-Sylvain, 2018).

\subsubsection{L'organisation du travail}

Le développement d'une compétence collective dans le télétravail demande une gestion particulièrement efficace dans l'utilisation des technologies de l'information et de la communication (TIC) ainsi que dans les écarts observés entre les activités prescrites et les activités réelles (Dupuich, 2011). Les erreurs répétées, mettant en cause la compétence de plusieurs personnes, résultent souvent de «malentendus dans la division du travail, du silence sur les désaccords [...] les causes individuelles et collectives s'articulent étroitement » (Livian, 
2010, p. 196). L'organisation du télétravail, selon Enel (2016), concerne différents éléments, dont les plus importants nous semblent être 1) le contrôle, 2) l'évaluation et 3) la formation et le recrutement.

Le contrôle du télétravail (plutôt invisible) amène des exigences peu réalistes, une perte de vue du temps que requiert la tâche et le manque de considération de la complexité de la tâche demandée (Racette et al., 2017). Une relation de confiance étroite doit donc être développée pour que le télétravail garde son efficacité, incarnée par le soutien organisationnel et social des dirigeants, des supérieurs et des collègues (St-Onge, Haines et Sevin, 2000). Ainsi, le contrôle, dans ce contexte, peut être interprété comme une surveillance excessive. Il peut s'agir de l'instauration d'un dispositif en temps réel de surveillance des courriels, de la vérification de rapports ainsi que d'un contrôle par les pairs, comparant le travail des télétravailleurs à celui de collègues en présentiel qui font partie d'une même équipe (Taskin, 2006).

Quant à l'évaluation du télétravailleur, elle peut reposer sur l'appréciation de la rapidité de ses réponses, de la capacité à répondre adéquatement aux questions qu'il reçoit, au nombre d'erreurs contenues dans les rapports et à la relation qu'il entretient avec les collègues. Dans ce dernier cas, il peut s'agir d'évaluer le tact dont font preuve les tuteurs dans leurs écrits, dont la présence d'émoticônes sourire, de formules de politesse, etc., et leur attitude en général. Toutefois, les critères d'accès au télétravail devraient être clairs et objectifs et faire l'objet d'un compromis avec les travailleurs (évaluation du travail, critères d'ancienneté, de performance, etc.) et l'information sur le télétravail devrait être donnée à tous les travailleurs (y compris à ceux qui ne sont pas des télétravailleurs).

Finalement, concernant les formations, elles peuvent être données à l'aide de capsules vidéo ou de rencontres en personne. Ces formations devraient fournir, entre autres, les consignes sur la façon de communiquer par courriel, en ce qui concerne par exemple les tournures de phrase, l'utilisation de majuscules ou de minuscules, la ponctuation et l'utilisation des «mercis » et des émoticônes. En matière de recrutement (Taskin et Tremblay, 2010), le supérieur hiérarchique devrait participer à la sélection des télétravailleurs pour que le travail s'inscrive dans une relation de confiance. Notons que lorsque le recrutement est fait à partir de références fournies par des employés, les travailleurs embauchés connaissent déjà quelqu'un de l'organisation et sont normalement plus impliqués.

\section{Des compétences nécessaires à une efficacité de tutorat}

Certains travaux font état des compétences particulières que doivent posséder les tuteurs pour assurer une efficacité dans l'encadrement des étudiants, mais également par rapport à la gestion du télétravail.

\subsection{Par rapport aux tuteurs}

Plusieurs auteurs s'entendent sur les compétences suivantes attendues dans l'encadrement des étudiants (Depover, 2013; Heiz, 2011; Pudelko, 2017; Sarpentier, 2015) :

1. Savoir organiser le travail d'encadrement;

2. Savoir intervenir sur les plans didactique, pédagogique, méthodologique, technologique et administratif;

3. Savoir évaluer les travaux, de façon constructive, rapide et justifiée; 
4. Savoir communiquer par écrit (principalement par courriel);

5. Savoir intervenir sur les plans socioaffectif, motivationnel et d'animation de groupe;

6. Savoir adopter une attitude professionnelle, de rigueur et d'honnêteté;

7. Savoir établir et maintenir une relation personnalisée, ouverte et de confiance;

8. Savoir respecter les étudiants.

Ces savoirs, bien que souhaitables dans toute situation d'emploi, s'avèrent particulièrement importants dans ce travail à distance des étudiants, des collègues tuteurs, des superviseurs immédiats et de tous les autres intervenants dans l'offre de cours en ligne. Toutefois, comme le souligne Depover (2013), bien que les tuteurs considèrent leur travail de première importance dans la formation des étudiants, leurs pratiques ne sont pas aussi soignées que l'organisation le souhaiterait, ce qui, selon les tuteurs, serait dû aux mauvaises conditions organisationnelles. Heiz (2011) appuie ce constat, précisant que les tuteurs doivent essentiellement ne compter que sur eux-mêmes.

Fernandez et al. (2014) et Rey et Sitnikoff (2006) soulèvent la difficulté du télétravailleur à maîtriser le temps et la portée dérégulatrice des TIC. Constatant l'ampleur du savoir-être que nécessite le télétravail, il n'est pas étonnant que, selon Enel (2016), les traits de personnalité recherchés du télétravailleur soient la maturité, l'entregent et la joie de vivre, son environnement le soutenant peu dans sa motivation et l'appréciation de son travail. Dans ce contexte particulier de télétravail, les compétences suivantes semblent lui être indispensables (Beauregard et al., 2019); Enel, 2016; Rey et Sitnikoff, 2006) :

1. Être autonome;

2. Être bon communicateur (l'écoute, le questionnement et la rétroaction);

3. Etre habile en informatique;

4. Être organisé;

5. Avoir l'esprit d'équipe;

6. Avoir des compétences en résolution de problèmes;

7. Savoir se réguler.

Lorsque l'employé travaille à son domicile, disposant de beaucoup moins de repères pour faire un bon travail, ses communications doivent être particulièrement soignées, se faisant surtout au moyen des courriels (Racette et al., 2018).

\subsection{Par rapport à la gestion du télétravail}

Afin de contrer l'isolement et de permettre la socialisation et le partage des connaissances des télétravailleurs, les solutions suivantes sont proposées (Taskin et Tremblay, 2010; Smith, Patmos et Pitts, 2018) :

1. Organiser des formations spécifiques pour les futurs télétravailleurs, entre autres sur la communication par courriel;

2. Échanger sur les expériences vécues entre les télétravailleurs;

3. Baliser le télétravail (modalités pratiques, etc.); 
4. Assurer un suivi régulier de la part du supérieur hiérarchique;

5. Formaliser des moments de coordination pour assurer la bonne transmission de l'information à tous;

6. Mettre au point des outils particuliers afin de permettre aux travailleurs de collaborer à distance (wiki et autres);

7. Mettre en place des tactiques de socialisation pour bâtir ou rebâtir la cohésion du groupe (consolidation d'équipe ou team building, etc.).

Selon Dupuich (2011), le transfert des connaissances peut être favorisé par le recours à un référentiel commun afin de guider l'action collective. De plus, des stratégies d'anticipation des conséquences des décisions prises permettraient de mieux appréhender les marges d'incertitude. Une bonne organisation des activités est cruciale. L'entraide, surtout au moyen des TIC, devrait permettre de résoudre les situations problématiques, ce qui nécessite le développement de la négociation et la solidarité dans la cueillette de l'information. Finalement, le partage de pratiques communes est essentiel, ce que favorisent les communautés de pratique, entre autres.

Pour contribuer tant au bien-être qu'à la performance des télétravailleurs, comme l'indiquent Poulsen et Ipsen (2017), il convient donc que les gestionnaires mobilisent à la fois des activités, soit des pratiques formelles et informelles, ainsi que des capacités telles que l'écoute, l'empathie, la confiance, de même que la valorisation de l'autonomie et de la responsabilisation. Toutefois, dans les écrits consultés, la mise en commun des pratiques dans les cours en ligne, entre les responsables des tuteurs et les tuteurs, est pour le moins timide.

\section{Conclusion}

Dans les cours en ligne, les processus sont fréquemment en changement. La gestion se fait surtout par essai-erreur, et le peu d'expérience acquise se transfère peu. Le télétravail est susceptible de favoriser la cristallisation des positions des équipes qui s'affrontent, chacune des équipes ayant besoin de l'autre, mais s'accusant mutuellement pour les problèmes qui ne manquent pas de se présenter. Cette gestion difficile du travail des télétravailleurs, comme le précisent Morissette et Bourhis (2019, p. 240), «peut générer des coûts indirects dus, par exemple, au manque de communication, à un leadership de style laisser-aller qui encourage les comportements individualistes, à une déconnexion... ». Aucune formation spécifique n'est normalement exigée pour ces tâches de tutorat, ni pour les gestionnaires de ce personnel en éducation. Quelques formations sont offertes aux tuteurs, mais non exigées, telles que le certificat au tutorat à distance offert par l'Agence universitaire de la Francophonie. Par rapport aux professeurs, comme le précisent Morissette et Bourhis (2019, p. 237), « il faut bien le dire, les professeurs ne sont pas formés pour assumer des responsabilités de gestion [...] enseigner un cours, mener une recherche et rédiger un article scientifique n'exigent pas les mêmes compétences que celles requises d'un gestionnaire ». Les spécialistes en éducation ne sont pas formés en gestion pour ce type de travail non plus. En plus des qualités que nécessite la gestion, la communication à distance présente des défis importants, différents de ceux de la communication en face à face (Pudelko, 2017).

La gestion des cours en ligne cherche toujours son équilibre. Il s'agit d'une forme de diffusion des enseignements relativement nouvelle pour les établissements. La tentation est grande d'apporter des changements surdimensionnés, qui s'accompagnent souvent d'effets pervers de résistances (Kourilsky, 2014). L'offre de cours en ligne semble souffrir de cette enflure, surtout 
dans le système d'encadrement des étudiants, dont les actions visent davantage l'homéostasie que le questionnement. Le manque de questionnements peut conduire au chaos, les actions peuvent se faire pour de mauvaises raisons ou simplement sans réelles raisons, ankylosant les systèmes (Carré, 2004/5). Pour éviter ces excès, une attention particulière doit être accordée à ce que vivent les acteurs impliqués, afin de leur offrir le soutien social et organisationnel dont ils ont besoin (St-Onge et al., 2000). Nous croyons, à l'instar de Morissette et Bourhis (2019), qu'une formation doit être offerte aux gestionnaires en éducation sur différents aspects, dont le leadership, la communication, la gestion des ressources humaines, les structures administratives et les règlements de l'université. Des communautés de pratique et des programmes de mentorat devraient être organisés. Des stratégies de rapprochement social et des zones de travail communes permettraient de développer une vie plus heureuse au travail, des cours plus performants et une meilleure expérience étudiante. De plus, les rôles et responsabilités de chacun devraient être mieux définis.

Cette tâche de gestion des tuteurs s'est ajoutée aux tâches des enseignants ou, encore, à celles des spécialistes en éducation en même temps que les cours en ligne se sont développés, mais sans une réelle prise en compte de leurs impacts. Ainsi, l'offre de cours en ligne de qualité demande des investissements importants. Aux coûts fixes de conception des cours et de leur mise en ligne s'ajoutent des coûts variables tels que ceux qui sont liés à l'encadrement des étudiants, à leur inscription ou à la tenue des examens (location de salles et embauche de surveillants). Mais, il existe également des coûts cachés absorbés par les responsables des cours, qui doivent trouver un équilibre entre leurs différentes tâches et les nouvelles attentes à leur égard. Il existe aussi des coûts cachés dus à des prises de décision dysfonctionnelles, à un dédoublement des tâches et à une perte d'économie d'échelle. Considérant le nombre de plus en plus important d'établissements ayant recours à un personnel affecté à l'encadrement, et de surcroit, par le télétravail, il apparaît essentiel d'examiner plus en profondeur les conséquences de ces choix organisationnels, pour le travailleur et pour l'établissement. Le CSE ( , p. 126) «met ainsi en garde les décideurs et acteurs universitaires contre une recherche d'économies au détriment de la qualité de la formation ».

\section{Références}

Baret, C. et Oiry, E. (2014). La gestion des compétences : quatre leçons pour surmonter les difficultés de la mise en œuvre dans le secteur public. Gestion, 39(2), 94-103. doi:10.3917/riges.392.0094

Barros, A. S. S. (2017). Subjective well-being (Sb) and burnout syndrome (BnS): Correlational analysis teleworkers education sector. Procedia - Social and Behavioral Sciences, 237, 1012-1018. https://doi.org/10.1016/j.sbspro.2017.02.144

Baruch, Y. et Nicholson, N. (1997). Home, sweet work: Requirements for effective home working. Journal of General Management, 23(2), 15-30. doi:10.1177/030630709702300202

Beauregard, T. A., Basile, K. A. et Canónico, E. (2019). Telework: Outcomes and facilitators for employees. Dans R. N. Landers (dir.), The Cambridge handbook of technology and employee behavior (p. 511-543). Cambridge, Royaume-Uni : Cambridge University Press. Manuscrit récupéré du répertoire BIROn - Birkbeck Institutional Research Online : http://eprints.bbk.ac.uk 
Béché, E. et Schneider, D. K. (2019). État des lieux de la recherche francophone sur les formations ouvertes et à distance. Distances et médiations des savoirs, 2019(27). https://doi.org/10.4000/dms.3910

Bloom, N., Liang, J., Roberts, J. et Ying, Z. J. (2014). Does working from home work? Evidence from a Chinese experiment. The Quarterly Journal of Economics, 130(1), 165-218. https://doi.org/10.1093/qje/q.ju032

Boboc, A. et Taboy, T. (2014). Numérique et transformations du monde du travail : vers de nouveaux équilibres. Récupéré du site Digital Society Forum : http://digital-societyforum.orange.com

Borges, J. P. F., Coelho, F. A. J., Faiad, C. et Rocha, N. F. (2014). Individual competences of distance education tutors. Educação e Pesquisa, 40(4), 935-951. https://doi.org/10.1590/s1517-97022014121642

Brindley, J. E. (2014). Learner support in online distance education: Essential and evolving. Dans O. Zawacki-Richte et T. H. Anderson (dir.), Online distance education: Towards a research agenda (p. 287-310). Edmonton, Canada : Athabasca University Press. https://doi.org/10.15215/aupress/9781927356623.01

Carré, P. (2004/5). Bandura : une psychologie pour le 16I siècle? Savoirs, 2004/5 (hors série), 9-50. https://doi.org/10.3917/savo.hs01.0009

Conseil supérieur de l'éducation (CSE). (2015). La formation à distance dans les universités québécoises : un potentiel à optimiser. Avis au ministre de l'Éducation, de l'Enseignement supérieur et de la Recherche. Québec, Canada : auteur. Récupéré du site de Bibliothèque et Archives nationales du Québec : http://numerique.banq.qc.ca

De la Rupelle, G., Casasus, S., Silva, F., Fatoux, F., Recorbet, L., Delassus, A., ... Chaussade, A. (2015). Du meilleur usage des outils de communication numérique dans les entreprises. Récupéré du site de l'Observatoire de la responsabilité sociétale des entreprises (ORSE) : http://orse.org

De Metz, N. et Bezuidenhout, A. (2018). An importance-competence analysis of the roles and competencies of e-tutors at an open distance learning institution. Australasian Journal of Educational Technology, 34(5), 27-43. https://doi.org/10.14742/ajet.3364

Depover, C. (2013). La place et l'importance du tutorat dans les nouveaux dispositifs de formation à distance. Dans P.-J. Loiret (dir.), Un détour par le futur. Les formations ouvertes et à distance à l'Agence universitaire de la Francophonie (p. 69-82). Récupéré du site Bibliothèque des savoirs en partage de l'Agence : http://bibliotheque.auf.org

Dupuich, F. (2011). L'émergence des compétences collectives, vers une gestion durable. Gestion 2000, 2011(28), 107-125. https://doi.org/10.3917/g2000.282.0107

Enel, L. (2016). Vers une meilleure compréhension de la construction du collectif de travail en contexte de télétravailleur : le cas d'une agence de placement de personnel (mémoire de maîtrise, Université du Québec à Montréal, Canada). Récupéré du répertoire Archipel : http://archipel.uqam.ca

Feldman, D. C. et Gainey, T. W. (1998). Patterns of telecommuting and their consequences: Framing the research agenda. Human Resource Management Review, 7(4), 369-388. doi:10.1016/S1053-4822(97)90025-5 
Fernandez, V., Guillot, C. et Marrauld, L. (2014). Télétravail et «travail à distance équipé »Quelles compétences, tactiques et pratiques professionnelles? Revue française de gestion, 2014/1(238), 101-118. https://doi.org/10.3166/RFG.238.101-118

Fisher, D. (1981). Communication in organizations. St. Paul, US : West Publishing Company.

Fonner, K. L. et Roloff, M. E. (2010). Why teleworkers are more satisfied with their jobs than are office-based workers: When less contact is beneficial. Journal of Applied Communication Research, 38(4), 336-361. https://doi.org/10.1080/00909882.2010.513998

Gajendra, R. S. et Harrison, D. A. (2007). The good, the bad and the unknown about telecommuting: Meta-analysis of psychological mediators and individual consequences. Journal of Applied Psychology, 92(6), 1524-1541. doi:10.1037/0021-9010.92.6.1524

Golden, T. D. (2006). The role of relationships in understanding telecommuter satisfaction. Journal of Organizational Behavior, 27(3), 319-340. https://doi.org/10.1002/job.369

Golden, T. D. et Veiga, J. F. (2005). The impact of extent of telecommuting on job satisfaction: Resolving inconsistent findings. Journal of Management, 31(2), 301-318. doi:10.1177/0149206304271768 Récupéré du profil de J. F. Veiga sur ResearchGate : http://researchgate.net/profile/John_Veiga

Golden, T. D., Veiga, J. F. et Dino, R. N. (2008). The impact of professional isolation on teleworker job performance and turnover intentions: Does time spent teleworking, interacting face-to-face, or having access to communication-enhancing technology matter? Journal of Applied Psychology, 93(6), 1412-1421. https://doi.org/10.1037/a0012722

Goodyear, P., Salmon, G., Spector, J. M., Steeples, C. et Tickner, S. (2001). Competences for online teaching: A special report. Educational Technology Research and Development, 49(1), 65-72. Récupéré du profil de P. Goodyear sur ResearchGate : http://researchgate.net/profile/Peter_Goodyear

Groupe AREVA. (2012). Accord collectif sur le développement de la qualité de vie au travail présenté au sein du groupe AREVA en France. Récupéré du site Dialogue social : http://dialogue-social.fr

Heiz, J. (2011). Formation à distance : relations entre responsables et tuteurs (mémoire de master, Université de Genève, Suisse). Récupéré du site du programme MALTT : http://tecfalabs.unige.ch/maltt

Heuel, E. et Feldmann, B. (2014). Quality standards for e-learning in vocational education and training: The certified European e-tutor. Dans L. Uden, Y.-H. Tao, H.-C. Yang et I. H. Ting (dir.), Proceedings of the 2nd International Workshop on Learning Technology for Education in Cloud (p. 93-100). Dordrecht, Pays-Bas : Springer. doi:10.1007/978-94007-7308-0_10

Imhoff, C. et Silva, F. (2016). Le développement personnel dans les organisations : entre modernité et post-modernité. Communication \& Management, 13(1), 63-77. doi:10.3917/comma.131.0063

Ismail, F. D., Hamsa, A. A. et Mohamed, M. Z. (2016). Factors influencing the stated preference of university employees towards telecommuting in international Islamic university Malaysia. Transportation Research Procedia, 17, 478-487. https://doi.org/10.1016/j.trpro.2016.11.097 
Kourilsky, F. (2014). Du désir au plaisir de changer. Le coaching du changement ( $5^{\mathrm{e}}$ éd.). Paris, France : Dunod.

Landry, S. et Gril, E. (2017). L'amélioration continue pour construire l'organisation apprenante. Gestion, 42(4), 96-99. doi:10.3917/riges.424.0096

Largier A. (2001). Le télétravail - Trois projets pour un même objet. Réseaux, 2001/2(106), 201-229. https://doi.org/10.3917/res.106.0201

Lédé, S. et Pelissier, C. (2019, avril). Trois facteurs de réussite dans la réutilisation des ressources numériques : le cas d'un cours de droit à l'université. Communication présentée au Colloque Ludovia\#CH, Haute école d'ingénierie et de gestion du canton de Vaud, Suisse. Récupéré de l'archive HAL : http://hal.archives-ouvertes.fr

Livian, Y.-F. (2010). Les organisations productrices d'incompétence. Revue internationale de psychologie, 39, 189-204. doi:10.3917/rips.039.0189

Marcotte, S. (2019). L'ère de la fragmentation. Dans P. Noreau et E. Bernheim (dir.), Devenir professeur (p. 277-85). Montréal, Canada : Presses de l’Université de Montréal.

McCloskey, D. W. et Igbaria, M. (2003). Does “out of sight” mean "out of mind"? An empirical investigation of the career advancement prospects of telecommuters. Information Resources Management Journal, 16(2), 19-34. Récupéré du profil de D. W McCloskey sur ResearchGate : http://researchgate.net/profile/Donna_Mccloskey3

McDowall, A. et Kinman, G. (2017). The new nowhere land? A research and practice agenda for the "always on" culture. Journal of Organizational Effectiveness: People and Performance, 4(3), 256-266. doi:10.1108/JOEPP-05-2017-0045 Manuscrit récupéré du répertoire de la Bedfordshire University : http://uobrep.openrepository.com

Morissette, L. et Bourhis, A. (2019). Concilier les rôles de professeur et de gestionnaire. Dans P. Noreau et E. Bernheim (dir.), Devenir professeur (p. 236-246). Montréal, Canada : Presses de l'Université de Montréal.

Poulsen, S. et Ipsen, C. (2017). In times of change: How distance managers can ensure employees' wellbeing and organizational performance. Safety Science, 100, 37-45. https://doi.org/10.1016/j.ssci.2017.05.002

Pudelko, B. (2017). L'encadrement dans les dispositifs en ligne ou hybrides en enseignement supérieur : des acteurs, des pratiques et des savoirs en émergence. Dans P. Pelletier et A. Huot (dir.), Construire l'expertise pédagogique et curriculaire en enseignement supérieur - Connaissances, compétences et expériences (p. 137-158). Québec, Canada : Presses de l'Université du Québec. Récupéré de http://puq.ca

Racette, N. et Bourdages-Sylvain, M.-P. (2019). Concevoir un cours à distance. Dans P. Noreau, et E. Bernheim (dir.), Devenir professeur (p. 113-124). Montréal, Canada : Presses de l'Université de Montréal.

Racette, N., Poellhuber, B. et Bourdages-Sylvain, M.-P. (2017). Quelles sont les caractéristiques de l'emploi et du travail des tuteurs en formation ouverte et à distance? Distances et médiations des savoirs, 2017(18). https://doi.org/10.4000/dms.1835

Racette, N., Poellhuber, B. et Bourdages-Sylvain, M.-P. (2018). L'organisation du travail entre les responsables de cours à distance et les tuteurs : un défi? Revue internationale des technologies en pédagogie universitaire, 15(1), 61-73. https://doi.org/10.18162/ritpu2018-v15n1-06 
Rey, C. et Sitnikoff, F. (2006). Travail à domicile et brouillage des frontières temporelles. Où va le temps de travail quand les activités professionnelles s'exercent à domicile? Loisir et société, 29(1), 101-116. https://doi.org/10.1080/07053436.2006.10707712

Roman, A. (2010). La banalisation du mail : l'ombre de Dionysos dans la communication organisationnelle. Société, 2010/1(107), 87-102. https://doi.org/10.3917/soc.107.0087

Rosa, H. (2019, 21-22 septembre). Les enseignants aux prises avec l'accélération du temps. Le Devoir, p. B9. Récupéré de http://ledevoir.com

Roy, N., Poellhuber, B. et Lefebvre, S. (2017). Les technologies : la compétence technopédagogique en enseignement supérieur. Dans P. Pelletier et A. Huot (dir.), Construire l'expertise pédagogique et curriculaire en enseignement supérieur. Connaissances, compétences et expériences (p. 117-135). Québec, Canada : Presses de l’Université du Québec. Récupéré de http://puq.ca

Sarpentier, C. (2015). La communication du tuteur en formation à distance : étude de cas unique analysant l'attrait de l'utilisation de l'enseignement explicite comme support dans les interventions écrites effectuées par des tuteurs de la TÉLUQ (mémoire de maîtrise, Université TÉLUQ, Canada). Récupéré du répertoire R-libre : http://r-libre.teluq.ca

Smith, S. A., Patmos, A. et Pitts, M. J. (2018). Communication and teleworking: A study of communication channel satisfaction, personality, and job satisfaction for teleworking employees. International Journal of Business Communication, 55(1), 44-68.

https://doi.org/10.1177/2329488415589101

St-Onge, S., Haines, V. Y. et Sevin, H. (2000). L'influence de l'appui du supérieur hiérarchique, des collègues et des dirigeants d'entreprise sur le succès du télétravail. Relations industrielles, 55(3), 414-50. https://doi.org/10.7202/051327ar

Taskin, L. (2006). Télétravail : les enjeux de la déspatialisation pour le management humain. Interventions économiques, 2006(34). https://doi.org/10.4000/interventionseconomiques.680

Taskin, L. (2010). La déspatialisation, enjeu de gestion. Revue française de gestion, 36(202), 61-76. https://doi.org/10.3166/RFG.202.61-76

Taskin, L. et Bridoux, F. (2010). Telework: A challenge to knowledge transfer in organizations. International Journal of Human Resource Management, 21(13), 2503-2520. https://doi.org/10.1080/09585192.2010.516600

Taskin, L. et Tremblay, D.-G. (2010). Comment gérer des télétravailleurs? Gestion, 35(1), 80-96. doi:10.3917/riges.351.008 Récupéré du répertoire R-libre : http://r-libre.teluq.ca

Tustin, D. H. (2014). Telecommuting academics within an open distance education environment of South Africa: More content, productive, and healthy? The International Review of Research in Open and Distributed Learning, 15(3). https://doi.org/10.19173/irrodl.v15i3.1770

Tutorat. (2003). Dans Le grand dictionnaire terminologique. Récupéré du site de l'Office québécois de la langue française : http://oqlf.gouv.qc.ca 